\title{
La innovación verde del modelo de negocio
}

\section{The green innovation of the business model}

\author{
Flavio Roberto Arroyo Morocho \\ Dely Nathalia Bravo Donoso \\ Carlos Santiago Buenaño Armas \\ Universidad Central del Ecuador, Ecuador \\ Freddy Lenin Villarreal Satama \\ Universidad Tecnológica Equinoccial, Ecuador \\ Autora para correspondencia:pgmejia@uce.edu.ec/pmejiagavilanez83@ hotmail.com \\ Fecha de recepción: 19 de diciembre 2017 - Fecha de aceptación: 10 de junio de 2018
}

Resumen: El presente artículo propone demostrar lo conveniente de una metodología de negocio verde y cómo puede permitir a las organizaciones para descubrir comercialmente interesantes oportunidades de negocios innovadores respetando el medio ambiente. Las empresas se enfrentan a la necesidad de pensar más estratégicamente sobre la sostenibilidad de su negocio. Metodologías gerenciales para negocios verdes pueden ayudar a transformar estos desafíos en nuevas oportunidades de mercado. El desarrollo y la aplicación de un modelo de negocio, que incorpora la sostenibilidad en todas las operaciones comerciales basadas en el pensamiento de ciclo de vida. Aplicar la una modelo de negocio verde puede ayudar a desarrollar a medida soluciones para satisfacer la creciente demanda del mercado ante los competidores. Esto permitirá acceder a nuevos segmentos de consumo, cadenas de suministro de grandes empresas y mercados internacionales. Además, una innovación verde sirve como un buen punto de partida para asociaciones con otras partes interesadas en la cadena de valor. Tal colaboración puede ayudar a su empresa a acceder a las redes de socios lo que facilita la penetración en el mercado y aumenta visibilidad de su marca.

Palabras Claves: innovación; modelo de negocio; ciclo de vida; eco-innovación; desarrollo sostenible; mercados globales

Abstract: This article allows for a green business methodology and how organizations can allow them to discover innovative business opportunities respecting the environment. Companies face the need to think more strategically about the sustainability of their business. Management methodologies for green businesses can help transform these challenges into new market opportunities. The development and application of a business model, which incorporates sustainability in all business operations in the life cycle. The application of the green business model can help develop solutions to meet the growing demand of the market against competitors. This allows access to new consumer segments, supply chains of large companies and international markets. In addition, a green innovation serves as a good starting point for partnerships with other stakeholders in the value chain. Such collaboration can help your company access partner networks that facilitate market penetration and increase the visibility of your brand.

Key Words: innovation; business model; life cycle; eco-innovation; sustainable development; global markets 


\section{Introducción}

El Modelo de Negocio Canvas es una herramienta a manera de plantilla de gestión estratégica para analizar los conceptos del modelo de negocio y explica cómo la compañía lo está lleva a cabo, cómo se crea valor para los clientes, la empresa y sus grupos de interés. Se compone de diferentes elementos como ingresos y costos, recursos, actividades, relaciones y redes internas y externas.

El Esquema de Innovación del Modelo de Negocio Verde (Green Business Model Innovation) conduce a una gran cantidad de diversas oportunidades de cambio en los modelos comerciales y aumentar su potencial para generar eco innovación sistémica, con el fin de hacer posible el objetivo de crecimiento verde del desacoplamiento absoluto de recursos.

Los modelos de negocios verdes que han ganado terreno deben garantizar el ahorro de energía para las empresas industriales y cobrar de acuerdo con los ahorros de energía logrados como resultado de sus instalaciones, también tiene el potencial de reducir los impactos ambientales de los productos químicos tóxicos.

Es así que, en la última parte, corresponde analizar a las principales barreras con las que se debe luchar en los modelos de ciclo de vida para que estos sean exitosos, además de estudiar la importancia que tiene el sector político, en la aplicación de normativas apara negocios verdes.

\section{Metodología}

\section{Desarrollo sostenible}

La sostenibilidad crea y mantiene las condiciones bajo las cuales los humanos y la naturaleza pueden existir en armonía, que permite cumplir los requisitos sociales, económicos y de otro tipo de las generaciones presentes y futuras (Li, Zhao, Zhang, Chen, \& Cao, 2017). La innovación verde es la creación o implementación de nuevos, o significativamente mejorados, productos (bienes y servicios), procesos, métodos de comercialización, estructuras organizacionales conduciendo a mejoras ambientales (OECD, 2009). El desarrollo sostenible es un concepto amplio que contiene una dimensión ecológica, económica y social y requiere sustanciales la innovación, y la innovación verde es una herramienta específica para lograr el desarrollo sostenible. La innovación verde se refiere a la innovación en tecnologías, productos, servicios, estructuras organizativas o modos de gestión adoptados empresas para lograr el desarrollo sostenible (Rennings, 2000). La innovación verde enfatiza la innovación hacia la sostenibilidad, contribuyendo a los esfuerzos en reducir las cargas ambientales. Las innovaciones sostenibles son invenciones que proporcionan un progreso esencial con respecto a las preocupaciones sociales, económicas y ecológicas (Rosca, Arnold, \& Bendul, 2017).

Para lograr una ventaja sostenible en el mercado globalizado, las empresas deben abordar tanto la innovación tecnológica e innovación administrativa, la adopción de nuevas estructuras organizativas o sistemas de gestión, mejorando así la producción y la gestión procesos para reducir los impactos ambientales negativos La innovación verde aplica el conocimiento científico ambiental y la tecnología para lograr un desarrollo armonioso de la economía y el medio ambiente en el proceso de producción. Estos esfuerzos incluyen desarrollar tecnologías y 
productos que ayudan a ahorrar energía y materias primas, utilizar la energía de manera eficiente e implementar empaques biodegradables (Kammerer, 2009).

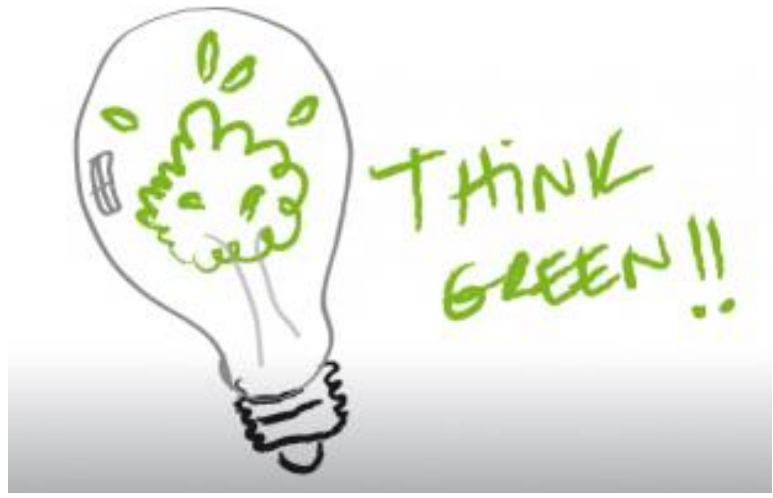

Figura 1. Pensamiento verde Fuente: (Nieto Gallego, 2012)

La innovación del modelo empresarial verde es la innovación en todo el ciclo empresarial, incluido el diseño, producción, suministro y uso final de productos comerciales que pueden reducir el costo de la energía en la sociedad y aumentar los beneficios para la empresa al mismo tiempo (Fei, Wang, Yang, Chen, \& Zhi, 2016). La innovación de negocios verdes se puede clasificar en tres categorías principales: innovación de productos verdes; innovación de procesos verdes; y verde gerencial innovación (Chen, Lai, \& Wen, The influence of green innovation performance on corporate advantage in Taiwan, 2006). La innovación verde está asociada positivamente con las empresas ventaja competitiva llamada competencias centrales verdes (Chen, The driver of green innovation and green image-green core competence, 2008). El producto que cumple con la demanda de los clientes y el requisito ambiental pueden destacarse en el mercado para obtener más beneficios para la compañía. Los negocios, como la parte más activa en la vida de la ciudad, cuya innovación verde está dirigida implementar programas de gestión ecológica de manera efectiva para mejorar el desempeño ambiental de una planta (Lenox, King, \& Ehrenfeld, 2000). Entonces, la innovación del modelo empresarial verde definitivamente puede conducir a una eco ciudad.

\section{¿Qué es la innovación verde del modelo de negocio?}

La innovación del modelo de negocio verde no siempre se debe a un cambio de una sola vez, con el objetivo de los efectos ecológicos y económicos, pero si el resultado de cambios continuos o la eficiencia gracias al modelo de negocio en el tiempo que finalmente termina siendo categorizado como innovación del modelo de negocio verde. Actualmente, existen varias empresas, quienes han implementado algunos métodos y estrategias tales como cradle to cradle, venta de productos o servicios ecológicos, modelos de incentivos, modelos del ciclo de vida entre otros, para crear nuevas oportunidades en el mercado. A continuación, se explican algunos de ellos:

- Los Modelos Incentivos: Se basan en cómo una empresa incentiva a sus consumidores de una manera en esa parte o toda la cadena de valor. Típicamente una empresa que mantiene la propiedad de un producto o que es pagada por incentivar su funcionalidad 
para producir, mantener y eliminar el producto de tal manera que toda la cadena de valor sea ecológica. Sin embargo, Estos modelos usan esquemas de incentivos y cambiado las estructuras de propiedad, así como la empresa entran en nuevos mercados en la cadena de valor. Ejemplos de estos modelos de incentivos son: Ventas funcionales, empresa de ahorro de energía, servicio de gestión de sustancias químicas, y finalmente el modelo diseñar, construir, financiar, operar.

- Modelos del Ciclo de Vida: Este modelo se puede dividir en varias categorías con respecto a qué parte y qué parte de la cadena de valor es eclipsada por el modelo. Si una empresa se enfoca en reverdecer toda la cadena de valor, existe una mayor posibilidad de que las acciones de la empresa se vean verdes en una perspectiva de ciclo de vida. La gestión de la cadena de suministro verde y la adquisición ecológica se centran en la parte superior de la cadena de valor mientras que la administración del producto, la responsabilidad extendida del productor y la gestión de la recuperación se centran en la cadena de valor descendente. Uno de los modelos usualmente utilizado en la última década, ha sido "cradle to cradle", además del modelo de Gestión de recuperación, seguido por "green supply chain management".

Las empresas diseñan y hacen cambios a su modelo de negocio estudiando los modelos de negocio de otras compañías, fortaleciendo aún más su posición en el mercado considerando los segmentos de clientes, su propuesta de valor, esquema de rentabilidad, diversas actividades y relaciones con los socios, entre otros, para cambiar el contenido de sus ofertas, su cadena de valor, reducir costos y riesgos y aumentar la rentabilidad.

El Modelo de Negocio Canvas desafía sistemáticamente la forma de hacer negocios y poder crear nuevas estratégicas, usándose para conceptualizar La Innovación del Modelo de Negocio Verde, una herramienta muy útil para descubrir los principales elementos de un modelo de negocio en relación con las prácticas ecológicas o sostenibles de las empresas.

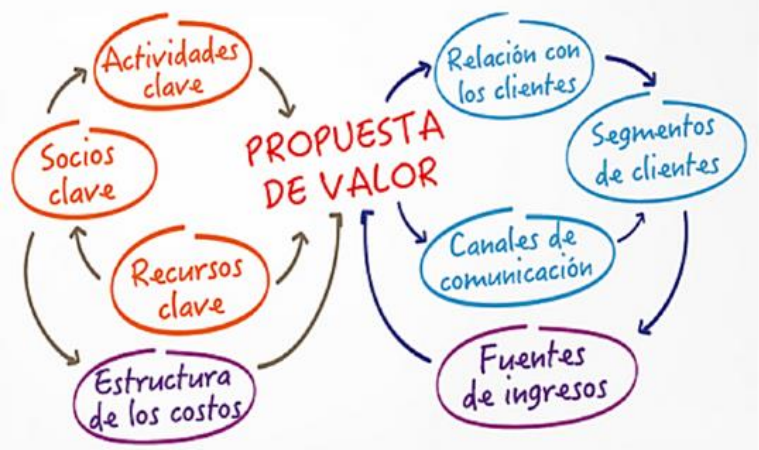

Figura 2. Modelo Canvas Fuente: (50Minutos.es, 2017)

La sostenibilidad corporativa ahora se encuentra en muchas compañías que juegan un papel integral en la formación de la misión o la fuerza motriz de una empresa. La Innovación del Modelo de Negocio Verde refleja el énfasis explícito del concepto en la reducción del impacto ambiental utilizando la eco innovación como respuesta de las prácticas corporativas hacia la 
sostenibilidad y el crecimiento ecológico, que se han materializado principalmente a un nivel de producto, pero algunas empresas han ampliado esta tendencia utilizándola para rediseñar sus procesos de producción o canales de distribución.

Muchas empresas emplean diferentes tipos de innovación en el modelo de negocio ecológico, respaldando un modelo de negocio verde más global y general, por ejemplo, Ventas funcionales (FS), Gestión de la Cadena de Suministro Ecológica (GSCM), Diseño de Cuna a la Cuna (C2C, Mecanismos de Devolución (TBM) y Simbiosis Industrial (IS).

\section{¿Qué hacen las compañías?}

Los autores Bisgaard, Henriksen, y Bjerre, en su libro "Gren Business Model Innovation" mencionan que los impulsores para una innovacion del modelo empresarial verde es una conciencia cada vez mayor del consumidor hacia la sostenibilidad. Ya que muchas compañías emplean la agenda verde como el promotor para alcanzar este tipo de modelos, sea cual sea el tamaño de la empresa y el sector, esto también como respuesta a los requerimientos del cliente, que está dispuesta a pagar y espera cada vez productos y servicios más responsables. (Bisgaard, Henriksen, \& Bjerre, 2012)

Otro impulsor es la oportunidad que tiene una empresa de diferenciar sus productos y servicios, dándole una ventaja competitiva. M. Ramos, menciona que la ventaja competitiva le da la excusa perfecta al cliente para hacer un esfuerzo por conseguirlo. (Ramos M. , 2015) También está el aumento de los costos de los recursos y riesgo del suministro, dando un nuevo enfoque a las empresas donde los desechos se utilizan como un recurso para el diseño de nuevos productos.

Así como existen impulsores también existen barreras. Bisgaard, Henriksen y Bjerre mencionan que estas son la falta de conocimientos y habilidades a lo largo de toda la cadena de valor de un producto o servicio, se pude mencionar en el desarrollo, producción y marketing. Además del gran costo de la nueva maquinaria y nuevos materiales o cambios que deben implementarse en el desarrollo de nuevos productos y diseño. (Bisgaard, Henriksen, \& Bjerre, 2012)

Para la transformación de elementos en modelos de negocios existen elementos de la innovación del modelo de negocio verde tales como el modelo comercial verde más ampliamente difundido dentro de los modelos de incentivos es Empresas de ahorro de energía (ESCO). Optimiza las operaciones de las empresas y los edificios públicos y, a cambio, se le paga de acuerdo con los ahorros logrados. Se especializa en la eficiencia del material y en la eficiencia del agua, que el material ahorre inversión en la empresa cliente. También en estos modelos, las compañías reciben una compensación en función de los ahorros de costos que obtienen para sus clientes.

Los servicios de gestión química (CMS) son un modelo comercial basado en un contrato estratégico a largo plazo, según el cual el proveedor de servicios de gestión de productos químicos acepta la responsabilidad de gestionar los productos químicos y se esfuerza por reducir los costes y riesgos asociados para el cliente. El proveedor de productos químicos y el usuario 
químico tienen el objetivo común de reducir los costos y las cantidades de productos químicos aplicados en los procesos del usuario.

Las empresas de diseño, construcción, finanzas, operación (DBFO) emprenden proyectos de construcción intensivos a largo plazo donde las finanzas privadas, la construcción, el servicio y mantenimiento se agrupan en un contrato a largo plazo de 20-30 años, que asigna riesgos y responsabilidades entre las partes. En este modelo de negocio, los contratos a largo plazo ofrecen incentivos para mejorar la calidad del proyecto de construcción, de modo que se reducen los costos del ciclo de vida.

Las empresas también realizan una Gestión de la cadena de suministro verde, donde puede ser definida como la inclusión de un pensamiento medio ambiental que incluye el diseño del producto, la selección y la fuente de los materiales, los procesos de fabricación, el envió del producto final a los consumidores y la gestión al final de la vida útil de tal producto, también es la combinación de las actividades de una empresa y el medio ambiente, así como la logística inversa, y destacó la importancia de implementar logística inversa para minimizar los residuos. También permite desarrollar un enfoque que promueve la sostenibilidad ambiental, eco diseño de producto gestión de proveedores y clientes, de tal manera que aparte de buscar la eficiencia y la productividad también se enfoque en el reciclaje, re-manufactura de productos y la eficiencia del diseño. Las empresas por medio de la GSCM estudian como el material reciclado puedan ser remplazados, sustituidos o convertirse en materia prima renovable para la reducción de costos en la producción masiva.

Retomar la gestión de los residuos es recuperar el uso de desechos de un producto a través de mecanismos, sus fabricantes, minoristas, consumidores y recicladores, los administradores establecen sistemas que les permitan recurar activos y reducir los costes de producción, también se crea un incentivo que permita conservar las propiedades del producto.

De la cuna de la cuna es una manera diferente de idear, diseñar y producir de forma que los elementos que conformen los productos puedan ser reutilizados y reciclados, donde los residuos que se producen en una empresa puede ser reutilizado en una esfera técnica o una esfera biológica, dado que está basado en tres principios que son residuo, energías renovables y la diversidad. Este concepto se desarrolló sobre todo en el lado material, y todavía hay potencial para una mayor innovación del modelo de negocio.

También está la simbiosis industrial es un enfoque de sistemas para una economía industrial más sostenible e integrado que identifica oportunidades de negocio que aprovechan lo subutilizando recursos como materiales, energía, agua, capacidad, experiencia, activos. En la simbiosis industrial tradicionalmente se compromete en un intercambio a través de las instalaciones compartidas. La pérdida de una empresa se convierte en la materia prima de otra, ambos beneficios medioambientales y de menor importancia se derivan de estos intercambios conocidos como "simbiosis industriales". 


\section{Resultados}

La medición de los resultados de las empresas a alcanzar después de transformar partes de sus modelos de negocio o la aplicación de otras nuevas no es tarea fácil. Primeros intentos de muchas empresas en verde modelo de negocio la innovación está dirigidas a un número limitado de líneas de productos o intentos iniciales en la venta de servicios de una manera nueva. Durante la prueba de las diferentes formas de hacer verde modelo de negocio la innovación foco no se coloca inicialmente en la forma de medir los resultados.

Es difícil aislar los indicadores específicos relacionados con los resultados de la innovación verde modelo de negocio, sobre todo si la innovación afecta sólo a una línea de productos donde las cifras de costes e ingresos no se descomponen en niveles tan detalladas. También es difícil medir los efectos de los cambios en los procesos en empresas tales como abastecimiento de recursos y materiales más ecológicos, ya que estos tipos de cambios puede tardar mucho tiempo antes de que se materialicen en un ahorro que se ven en la línea inferior. Sin embargo, todas las empresas ver verde modelo de negocio la innovación como una manera de crear impactos ambientales positivos, más innovación y beneficio económico.

\section{Política para promover la innovación del modelo de negocio verde}

Varias empresas realizan mejoras ambientales basadas en el modelo de negocio ecológico, realizando mediciones minuciosas o simplemente realizando estimaciones. Los impactos importantes que se han reducido implementando el modelo de negocio verde son el consumo de materias primas, emisiones de gases efecto invernadero, consumo de energía, desechos y aumentado el reciclaje, productos químicos tóxicos y agua. El modelo de negocios donde se ve el mayor efecto es en el modelo comercial de la cuna a la cuna, donde todas las empresas implementaron una reducción en productos químicos tóxicos en sus productos y procesos.

Las políticas para el modelo de negocio verde deben ser innovadoras y creativas, brindando seguimiento ya que si se deja a la deriva este modelo no puede funcionar y necesitaría de otro tipo de políticas para subsistir. Se han identificado varias políticas que apoyan la innovación del modelo de negocio verde, que han ayudado a crear una imagen de dónde los gobiernos han intentado apoyar a las empresas, y donde aún no se han hecho intentos, una política solo puede considerarse buena si hay resultados que mostrar y si estos son positivos puede promoverse a su uso en todo el mundo evaluando los costos de implementación. Las políticas que se implementen a nivel internacional deben adaptarse a la región donde se la quiera asentar.

Existen modelos de negocios con sus respectivas políticas como, por ejemplo:

- Empresas de ahorro de energía (ESCO) es el único modelo funcional de ventas que ha logrado atención debido a que se requiere reducir la cantidad de energía consumida en la producción.

- CMS (Chemical management services) gestión de servicios químicos trata de eliminar químicos tóxicos en la industria lo cual es un fenómeno global, esta política debería 
alentar el uso de sustancias químicas alternativas no generan residuos químicos como Green Chemistry42.

Cuando se trata de políticas para los modelos del ciclo de vida, se identificaron las iniciativas de políticas para dos categorías principales de elementos del modelo de negocio. Políticas como la reutilización y reciclaje de aparatos electrónicos, es el primer paso para considerar que sucede con estos aparatos cuando ya se vuelven obsoletos; otra como, de cuna a la cuna la cual se centra en la prevención de residuos. Si bien algunos países han desarrollado una política que se dirige a modelos comerciales específicos, no parece haber muchos países que hayan implementado iniciativas de políticas que promuevan el uso de la Innovación del Modelo de Negocio Verde a nivel general. La única iniciativa de política relevante que hemos podido identificar es el Fondo de Innovación Empresarial de Dinamarca.

Es así que según (Bisgaard, Henriksen, \& Bjerre, 2012) indican las principales inconvenientes que surgen al utilizar los diferentes modelos de ciclo de vida, es así que entre los principales tenemos a los modelos de abastecimiento de cadenas verdes, TBM, de la cuna a la cuna y el modelo de simbiosis industrial; todos estos modelos tienen sus ventajas y desventajas, lo primordial es saber cuándo efectuar cada uno sortear sus obstáculos y lograr así un mínimo impacto en los ámbitos ecológico, social y monetario.

Por otro lado la importancia del sector político en generar normativas para negocios verdes es de gran importancia (Descola \& Pálsson, 2001) tanto que su participación es indispensable ya sea en licitaciones, creación de captadoras y recicladoras, realizando asociaciones claves, etc.

Al trabajar con el concepto "verde" se cumple con la responsabilidad social. Esto puede ayudar a promocionar un negocio y obtener beneficios de mercadeo con una proyección incalculable. Una solución a muchas de las barreras para la innovación en un modelo de negocio verde es la creación de asociaciones que ayuden impartiendo cursos y financiando costos.

\section{Conclusiones}

Cada día son más los emprendedores que se deciden por iniciar un negocio verde, pues la protección del medio ambiente cala cada vez más hondo entre los consumidores. Estos, a su vez, demandan productos y servicios que no sólo cumplan con la calidad de siempre, sino que, además, estén comprometidos con el planeta y sean respetuosos con él. La ventaja más sobresaliente de un negocio verde es la reducción de costos de operación, lo que también se puede traducir en enormes beneficios netos para tu empresa. Analiza bien este tema, pues te permitirá ahorrar mucho dinero para futuros proyectos.

Un factor importante para tener éxito con La Innovación del Modelo de Negocio Verde es crear una cultura de empresa en la que los empleados comprendan qué significa la sostenibilidad para la compañía, basándose en un enfoque ecológico a la hora de ofrecer sus productos y servicios al mercado. El cliente es el principal impulsador para la innovación en un modelo de negocio verde, debido a sus requerimientos cada vez mayores enfocados en la sostenibilidad y cuidado al medio ambiente, incentivan y fomentan a que las industrias se acoplen a estos requerimientos, y desarrollen nuevos productos y servicios de tal manera que les provea una ventaja competitiva. En este proceso se enfrentan a barreras que radican principalmente en la 
falta de conocimiento, hábitos de compra y costos de implementación. Una correcta asociación de las empresas dentro de modelos de negocios, facilita el manejo de los desechos y a la vez permite que exista una responsabilidad compartida de donde vienen las materias primas utilizadas, reduciendo la cantidad necesitada de estos insumos, así como una correcta disposición de los residuos.

La GSCM es una estrategia la cual se fundamente en un concepto medio ambiental donde el punto principal es mejorar la línea de producción de un producto aprovechando sus residuos y el reciclaje generado a través de la reutilización de los residuos del producto aprovechando la materia prima y volviéndola a incluir en la producción

Al implementar políticas para el modelo de negocio verde se busca un mejor desempeño de las empresas en materia ambiental, algunas de estas aún están en proceso de elaboración, por lo tanto, aunque las ideas parecen buenas, no es posible saber si alcanzarán los objetivos deseados. Las políticas ayudarán a las empresas a actuar con mayor responsabilidad social frente a los impactos que se generan al medio ambiente en la producción de bienes.

Finalmente, en cuanto a las políticas, se puede aseverar que los mandatarios políticos son pieza clave en la creación de normativas que impulsen la sustentabilidad en sus territorios, así se puede tomar de ejemplo a países como Noruega, Irlanda, Dinamarca, Islandia, Reino Unido entre otros, por su gran aporte a las políticas de innovación para modelos de negocios verdes ahora son referentes a nivel mundial en temas ambientales y ecológicos.

\section{Bibliografía}

50Minutos.es. (2017). El modelo Canvas: Analice su modelo de negocio de forma eficaz. España: 50Minutos.es.

Bisgaard, T., Henriksen, K., \& Bjerre, M. (2012). Green Business Model Innovation. Nordic Innovation.

Chen, Y.-S. (2008). The driver of green innovation and green image-green core competence. Journal of business ethics, 531-543.

Chen, Y.-S., Lai, S.-B., \& Wen, C.-T. (2006). The influence of green innovation performance on corporate advantage in Taiwan. Journal of Business Ethics, 331-339.

Descola , P., \& Pálsson, G. (2001). Naturaleza y sociedad: perspectivas antropológicas. Mexico: Eds.

EOI. (3 de Septiembre de 2015). ¿Qué es la innovación? . Obtenido de http://www.eoi.es/blogs/redinnovacionEOI/2015/09/03/que-es-la-innovacion/

Fei, J., Wang, Y., Yang, Y., Chen, S., \& Zhi, Q. (2016). Towards eco-city: the role of green innovation. Energy Procedia, 165-170. 
Kammerer, D. (2009). The effects of customer benefit and regulation on environmental product innovation.: Empirical evidence from appliance manufacturers in Germany. Ecological Economics, 2285-2295.

Lenox, M., King, A., \& Ehrenfeld, J. (2000). An Assessment of Design-for-Environment Practices in Leading US Electronics Firms. Interfaces, 83-94.

Li, D., Zhao, Y., Zhang, L., Chen, X., \& Cao, C. (2017). Impact of quality management on green innovation. Journal of Cleaner Production, 462-470.

Nieto Gallego, E. (2012). Ecoinnovación en procesos industriales. Madrid: EOI.

OECD. (2009). Sustainable Manufacturing and Eco-innovation: Towards a Green Economy. París: OECD.

Ramos, M. (16 de Junio de 2015). Stratgia. Obtenido de La ventaja competitiva sostenible en el tiempo y cómo diferenciarse: http://www.stratgia.com/blog/la-ventaja-competitivasostenible-y-como-diferenciarse

Rennings, K. (2000). Redefining innovation - eco-innovation research and the contribution from ecological economics. Ecological Economics, 319-332.

Rosca, E., Arnold, M., \& Bendul, J. C. (2017). Business models for sustainable innovation - an empirical analysis of frugal products and services. Journal of Cleaner Production, S133S145.

Tanja Bisgaard, K. H. (Octubre de 2012). Green Business Model Innovation. Obtenido de NORDIC INNOVATION: http://www.nordicinnovation.org/Global/_Publications/Reports/2012/2012_12\%20Green $\% 20$ Business\%20Model\%20Innovation_Conceptualisation\%20next\%20practice\%20and $\%$ 20policy_web.pdf 